\title{
Cocoon Syndrome: A Rare Entity
}

\author{
Sonia Salas Diaz ${ }^{\mathrm{a}}$, Manuel Jesus Saavedra Chacon ${ }^{\mathrm{a}}$, \\ Rafael Trujillo Vilchez ${ }^{\mathrm{b}, \mathrm{c}}$
}

\begin{abstract}
Sclerosing encapsulating peritonitis (SEP) is an uncommon condition characterized by the encasement of small bowel due to a thick fibrotic coverage. This entity is usually related to several diseases, highlighting among them the peritoneal dialysis, and less frequent is the primary or idiopathic peritoneal sclerosis or cocoon syndrome. SEP is clinically variable, depending on the severity and duration of the disease, the underlying causes and the patient's immune status. The most common presentation is intestinal obstruction. It is rarely presented as a complication such as enterocutaneous fistulas, intestinal necrosis and malnutrition. Intestinal perforation is quite uncommon in patients with SEP. We present a case of cocoon syndrome in a 67-year-old man and describe his clinical onset, radiology and anatomical pathology findings.
\end{abstract}

Keywords: Obstructive bowel; Surgery; Cocoon syndrome

\section{Introduction}

The importance of this case relies on the very low frequency of this disease. The difficulty of the diagnosis is the common presentation of the condition as a chronic abdominal unspecific pain and weight loss which makes it difficult to identify clinically unless the patient develops a bowel obstruction. It is also exceptional with the presentation of sclerosing encapsulating peritonitis (SEP) as a primary entity [1].

\section{Case Report}

A 67-year-old male patient was referred from the gastroenterology department to the general surgery department for an exploratory laparotomy with biopsy after several episodes of bowel subclusion for the last year without finding a mechanic cause to justify the symptoms. In his personal history, we can

Manuscript submitted August 31, 2017, accepted October 31, 2017

apunta de Europa Hospital, Algeciras, Spain

bVithas Xanit International Hospital, Benalmadena, Spain

${ }^{\mathrm{c} C o r r e s p o n d i n g ~ A u t h o r: ~ R a f a e l ~ T r u j i l l o ~ V i l c h e z, ~ V i t h a s ~ X a n i t ~ I n t e r n a t i o n a l ~}$

Hospital, Benalmadena, Spain. Email: rafaeltruj@gmail.com

doi: https://doi.org/10.14740/jcs334e highlight mellitus diabetes treated with insulin and gastric ulcer diagnosed 30 years ago treated with proton pump inhibitors. On exploration, the patient presented with severe proteincalorie malnutrition, hyporexia, vomiting every 3 days and a weight loss of $23 \mathrm{~kg}$.

\section{Investigations}

Among the diagnostic tests that were performed with the intent to diagnose the patient, we wanted to focus on the gastroesophageal studies that revealed a generalized dilatation of the first part of the bowel and a slow gastric emptying. Computerized tomography (CT) presented an important duodenal, gastric and jejunal distension with plenty of content. The intestinal magnetic resonance imaging (MRI) emphasized a dilated gastric chamber and duodenum (Fig. 1).

\section{Differential diagnosis}

The differential diagnosis included systemic lupus erythematosus, tuberculosis, sarcoidosis, and amyloidosis.

\section{Treatment}

Finally, the patient underwent surgery and an exploratory laparotomy was made, showing a constrictive peritonitis caused by a whitish fibrotic tissue that embraced all the small bowel with dilatation of the stomach and duodenum. All the constrictive tissue was removed and a $5 \mathrm{~cm}$ long biopsy of small bowel was taken (Fig. 2).

\section{Outcome and follow-up}

The pathological anatomy of the biopsies taken showed a generalized chronic inflammatory infiltrate with deposits of collagen with hyaline areas and poor cellularity in the outer layers of the intestine. It is concluded that the diagnosis was peritoneal fibrosis.

After a multidisciplinary hospital session where the case was presented, it was concluded that the definitive diagnostic was primary sclerosing peritonitis or cocoon syndrome.

The postoperative course was favorable and the patient was discharged 5 days after surgery with a corticoid treatment based on the current guidelines to avoid recurrence. In the suc- 


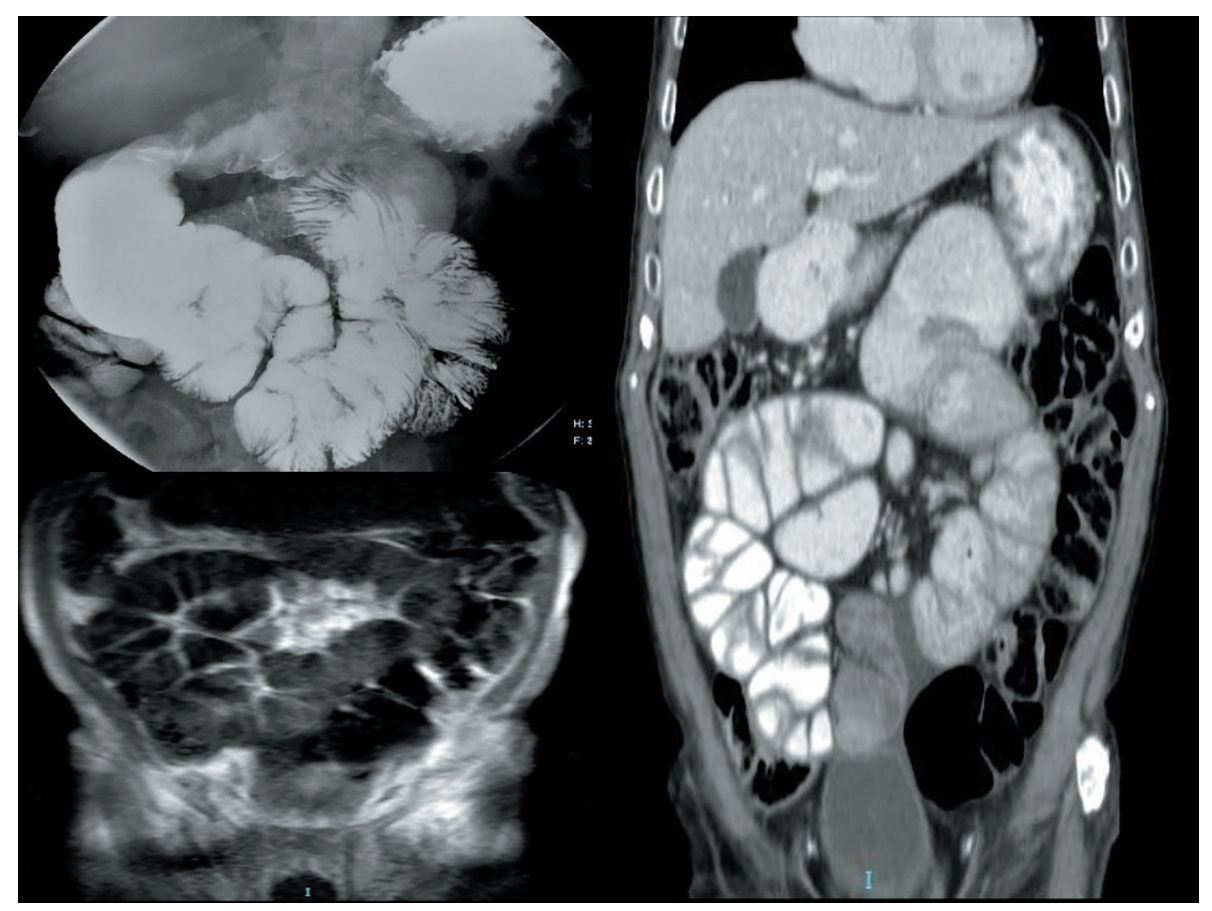

Figure 1. Radiological sequence was done to our patient. Oesophageal-gastric-duodenal transit shows generalized dilatation of small bowel loops. Computed tomography (CT) presents significant gastric and duodenal distension (up to $85 \mathrm{~mm}$ ).

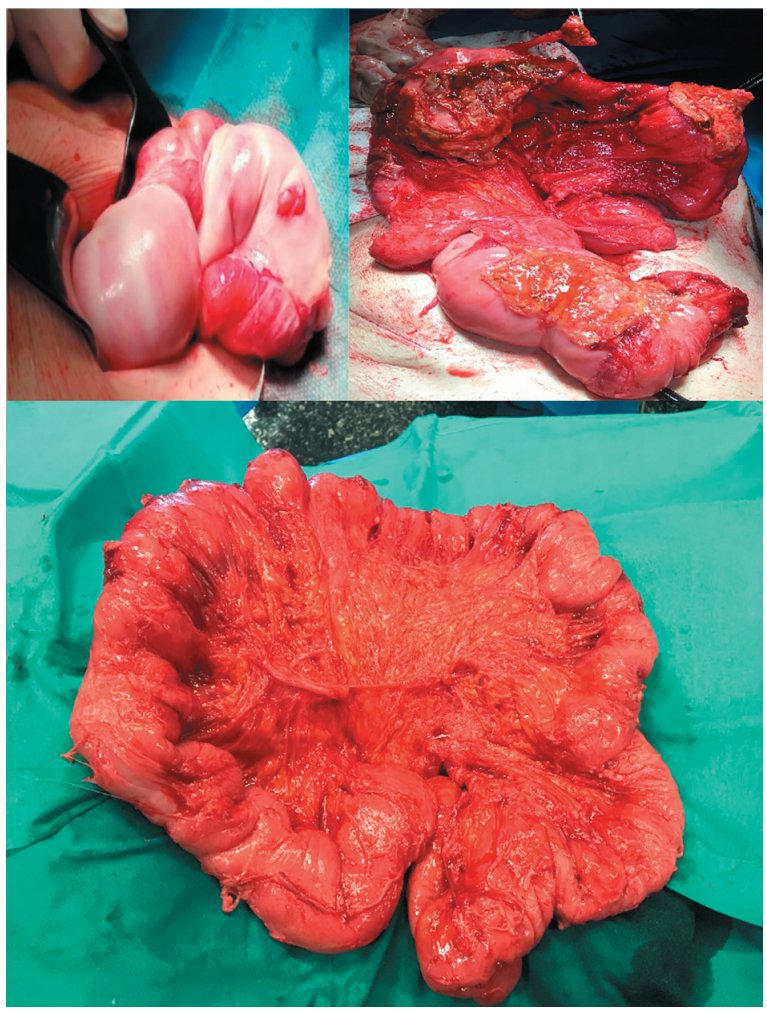

Figure 2. The patient underwent surgery and an exploratory laparotomy was made, showing a constrictive peritonitis caused by a whitish fibrotic tissue that embraced all the small bowel with dilatation of the stomach and duodenum. All the constrictive tissue was removed and a $5 \mathrm{~cm}$ long biopsy of small bowel was taken. cessive follow-ups, the patient showed a considerable weight gain with the improvement of the performance status.

\section{Discussion}

Achieving accurate diagnosis in affected patients is extremely difficult. The preoperative study should rule out secondary causes of SEP such as tuberculosis, Parkinson's disease, systemic lupus erythematosus, post-surgical adhesion syndrome, etc. The vast majority of cases are diagnosed based on intraoperative findings. Radiographs may show diffuse air-fluid levels and dilatation of the small intestine in the form of loops [2]; however, these findings are not specific. Barium studies usually show bowel loops that accumulate in the center of the abdomen, and this finding is called "sign of cauliflower or accordion". In addition, a slow transit can help the diagnosis as it appeared in our case. CT may show intestinal obstruction, ascites, localized collections, peritoneal or mesenteric thickening, calcifications and lymphadenopathy [2]. The findings of the MRI enterography are usually similar to CT. Surgery has a relevant role in many cases, in order to confirm the diagnosis with a biopsy after ruling out other secondary causes [3].

There is no consensus regarding the optimal treatment in patients with SEP, since it is a very rare entity. In patients with mild symptoms, conservative management including bowel rest, nasogastric tube decompression and nutritional support may be considered the most appropriate therapeutic option [4], as a medical treatment also included the use of drugs such as tamoxifen, steroids, colchicine, and azathioprine $[2,5]$. In 
those patients with severe signs of intestinal obstruction, the most appropriate procedure consists of adhesiolysis and excision of the membrane that encapsulates the intestinal surface, with a low recurrence risk [1] and satisfactory long-term results $[1,6,7]$.

A limited number of case reports have successfully described membrane excision and laparoscopic adhesiolysis [8, 9]. To avoid complications, such as leakage of anastomosis and short bowel syndrome, in patients with sclerosing peritonitis, intestinal resection is indicated only when necrosis develops [10]. Resection is often unnecessary and, when performed without a clear indication, it may increase patient morbidity and mortality $[1,4]$. The administration of an antifibrogenic and anti-inflammatory agents during the postoperative period in patients in which the encapsulating membrane cannot be completely excised appears to be beneficial [2].

\section{Learning points/take home messages}

A biopsy is mandatory in the patients with a long history of abdominal subclusion when all the radiological findings do not demonstrate a cause.

Exploratory laparotomy is the best option to remove the whole cover of the bowel.

Medical treatment may be used when the patient's symptoms are mild or in conjunction with surgical treatment to avoid recurrence or in cases of incomplete excision of fibrosis.

\section{References}

1. Li N, Zhu W, Li Y, Gong J, Gu L, Li M, Cao L, et al.
Surgical treatment and perioperative management of idiopathic abdominal cocoon: single-center review of 65 cases. World J Surg. 2014;38(7):1860-1867.

2. Habib SM, Betjes MG, Fieren MW, Boeschoten EW, Abrahams AC, Boer WH, Struijk DG, et al. Management of encapsulating peritoneal sclerosis: a guideline on optimal and uniform treatment. Neth J Med. 2011;69(11):500507.

3. Akbulut S, Yagmur Y, Babur M. Coexistence of abdominal cocoon, intestinal perforation and incarcerated Meckel's diverticulum in an inguinal hernia: A troublesome condition. World J Gastrointest Surg. 2014;6(3):51-54.

4. Kaur R, Chauhan D, Dalal U, Khurana U. Abdominal cocoon with small bowel obstruction: two case reports. Abdom Imaging. 2012;37(2):275-278.

5. Cornelis T, Oreopoulos DG. Update on potential medical treatments for encapsulating peritoneal sclerosis; human and experimental data. Int Urol Nephrol. 2011;43(1):147156.

6. Singh B, Gupta S. Abdominal cocoon: a case series. Int J Surg. 2013;11(4):325-328.

7. Kirshtein B, Mizrahi S, Sinelnikov I, Lantsberg L. Abdominal cocoon as a rare cause of small bowel obstruction in an elderly man: report of a case and review of the literature. Indian J Surg. 2011;73(1):73-75.

8. Gadhire M, Singh MB, Jshi M. Abdominal cocoon syndrome. JEMDS. 2013;2:1857-1861.

9. Meshikhes AW, Bojal S. A rare cause of small bowel obstruction: Abdominal cocoon. Int J Surg Case Rep. 2012;3(7):272-274.

10. Tannoury JN, Abboud BN. Idiopathic sclerosing encapsulating peritonitis: abdominal cocoon. World J Gastroenterol. 2012;18(17):1999-2004. 